\title{
A comparison of direct reconstruction algorithms in pro- ton computed tomography
}

\author{
F. Khellaf ${ }^{1}$, N. Krah ${ }^{1,2}$, J. M. Létang ${ }^{1}$, Charles-Antoine Collins-Fekete ${ }^{3}$, S. Rit ${ }^{1}$ \\ ${ }^{1}$ University of Lyon, INSA-Lyon, Université Claude Bernard Lyon 1, UJM-Saint Etienne, CNRS, Inserm, CREATIS UMR \\ 5220, U1206, F-69373, LYON, France. \\ ${ }^{2}$ Institut de Physique des Deux Infinis de Lyon, UMR 5822, F-69622, Villeurbanne, France. \\ ${ }^{3}$ Department of Medical Physics and Biomedical Engineering, University College London, Gower Street, London, UK. \\ feriel.khellaf@creatis.insa-lyon.fr
}

\begin{abstract}
Several direct algorithms have been proposed to take into account the non-linear path of protons in the reconstruction of a proton $\mathrm{CT}(\mathrm{pCT})$ image. This paper presents a comparison between five of them, in terms of spatial resolution and relative stopping power (RSP) accuracy. Our comparison includes (1) a distance-driven algorithm extending the filtered backprojection to non-linear trajectories (DD), (2) an algorithm reconstructing a pCT image from optimized projections (ML), (3) a backproject-then-filter approach using a 2D cone filter (BTF), (4) a differentiated backprojection algorithm based on the inversion of the Hilbert transform (DBP), and (5) an algorithm using a 2D directional ramp filter (DR). We have simulated a single tracking pCT set-up using Geant4 through GATE, with a proton source and two position, direction and energy detectors upstream and downstream from the object. Tracker uncertainties were added on the position and direction measurements. A Catphan 528 phantom and a spiral phantom were simulated to measure the spatial resolution and a Gammex 467 phantom was used for the RSP accuracy. Each proton's trajectory was estimated using a most likely path (MLP) formalism. The spatial resolution was evaluated using the frequency corresponding to an MTF of $10 \%$ of its peak value and the RSP accuracy using the mean values in the inserts of the Gammex phantom. In terms of spatial resolution, it was shown that, for ideal trackers, the DR and BTF methods offer a slightly better resolution since each proton is directly binned in the image grid according to its MLP. However, all methods but the ML show comparable resolution when using realistic trackers. Regarding the RSP, three algorithms (DR, DD and BTF) show a mean relative error inside the inserts about $0.1 \%$. As the DR and BTF methods are more computationally expensive, the DD - which allows the same spatial resolution in realistic conditions and the same accuracy - and the DBP — which has a fairly good accuracy $(<0.2 \%)$ and allows reconstruction from truncated data - can be used for a reduced reconstruction time.
\end{abstract}

Keywords: proton $\mathrm{CT}$ reconstruction, spatial resolution, direct algorithms

\section{INTRODUCTION}

Proton computed tomography (pCT) has been developed to reduce particle range uncertainties in hadron therapy treatment planning (Schaffner et al., 1998; Yang et al., 2012; Paganetti, 2012). While current methods are based on a calibration between X-ray CT numbers and proton relative stopping power (RSP), pCT allows for a direct reconstruction of the RSP map and therefore a reduction of range uncertainties (Arbor et al., 2015). Although pCT provides a better estimation of the RSP, it suffers from poor spatial resolution, compared to X-ray CT, due to the stochastic nature of the non-linear proton paths. Disregarding nuclear interactions, a proton trajectory is a random combination of many small angular deflections due to multiple Coulomb scattering (MCS). It has been shown that these trajectories can be estimated using a most likely path (MLP) formalism (Williams, 2004; Schulte et al., 2008), a cubic spline path (Li 
et al., 2006; Fekete et al., 2015) or a polynomial model (Krah et al., 2019), and including these estimates in the reconstruction can substantially improve spatial resolution.

The inverse problem in $\mathrm{pCT}$ is similar to that in X-ray CT as it consists in reconstructing an image from line integrals. What makes it different are the non-linear integration lines representing the proton path estimates. Several reconstruction methods incorporating a proton path estimate have been proposed for pCT (Li et al., 2006 Penfold et al., 2010 Rit et al., 2013, Poludniowski et al., 2014; Hansen et al., 2014a; Hansen et al., 2014b; Rit et al., 2015; CollinsFekete et al., 2016; Hansen et al., 2016). However, since there is no exact analytical solution to invert proton integration paths yet, all proposed algorithms are heuristic solutions. An often used direct reconstruction method is the distance-driven binning algorithm, which generalizes the filtered backprojection algorithm to non-linear paths. Other direct algorithms invert the backprojection and filtering steps as it would be more fitting to list-mode proton data. Alongside direct reconstructions, various iterative algebraic methods were proposed, from the classical ART (Gordon et al., 1970) to total variation superiorization algorithms. Iterative algorithms have advantages, e.g. including priors to improve image reconstruction (Hansen et al., 2014a), however, their computational cost is generally an order of magnitude larger than direct methods. Hansen et al. (2016) compared the distance-driven method and three iterative algorithms to reconstruct low dose pCT images. The authors conclude that the distance-driven algorithm allows to reconstruct high-quality images while being at least 3 and up to 22 times faster than iterative algorithms.

However, no comparison between direct reconstruction methods was reported in the literature. The purpose of this paper is to compare five different direct algorithms based on spatial resolution as well as RSP accuracy. Although some of the direct methods are quite close, they use different kinds of approximations which could impact spatial resolution and/or RSP accuracy. For instance, as stated previously, backproject-filter approaches fit the list-mode data more naturally and might give a better spatial resolution, but some of these methods also require the computation of correction terms that might degrade the RSP accuracy. By comparing these methods, this work aims to select the algorithm giving the best spatial resolution while keeping a high RSP accuracy (relative error about 0.1\% to 0.5\% (Penfold et al., 2009; Poludniowski et al., 2014)) with a reasonable computational cost.

\section{MATERIALS AND METHODS}

\subsection{Reconstruction in proton CT}

The aim of pCT reconstruction is to build an RSP map from the protons' energy loss. For a given proton $i$, the line integral of the RSP is related to the energy loss using

$$
\mathrm{WEPL}_{i} \equiv \int_{\boldsymbol{\Gamma}_{i}} \operatorname{RSP}(\boldsymbol{x}) \mathrm{d} \boldsymbol{x} \approx \int_{E_{i}^{\text {out }}}^{E_{i}^{\text {in }}} \frac{\mathrm{d} E}{S_{\text {water }}(E)}
$$

where $\boldsymbol{\Gamma}_{i} \subset \mathbb{R}^{3}$ is the proton path, $\operatorname{RSP}(\boldsymbol{x})$ is the stopping power relative to water at position $\boldsymbol{x} \in \mathbb{R}^{3}, E_{i}^{\text {in }}$ and $E_{i}^{\text {out }}$ are the entrance and exit proton energies, and $S_{\text {water }}(E)$ is the stopping power of water for energy $E$. It is assumed that the RSP is energy independent (less than $0.7 \%$ variations in the 80-300 MeV energy range for surrogate human tissues Arbor et al., 2015) for the equality to be valid. The integral corresponds to the water-equivalent path length (WEPL).

One of the proposed set-ups for pCT tracks each proton individually and measures its position and direction before and after traversing the object, as well as its energy loss (Johnson, 2018). It is possible to get each proton's WEPL from the energy measurement using the right-hand side of Equation 1. Its MLP is computed from the position and angle information. The pCT reconstruction problem consists in using each proton's WEPL and MLP to recover the RSP map. 

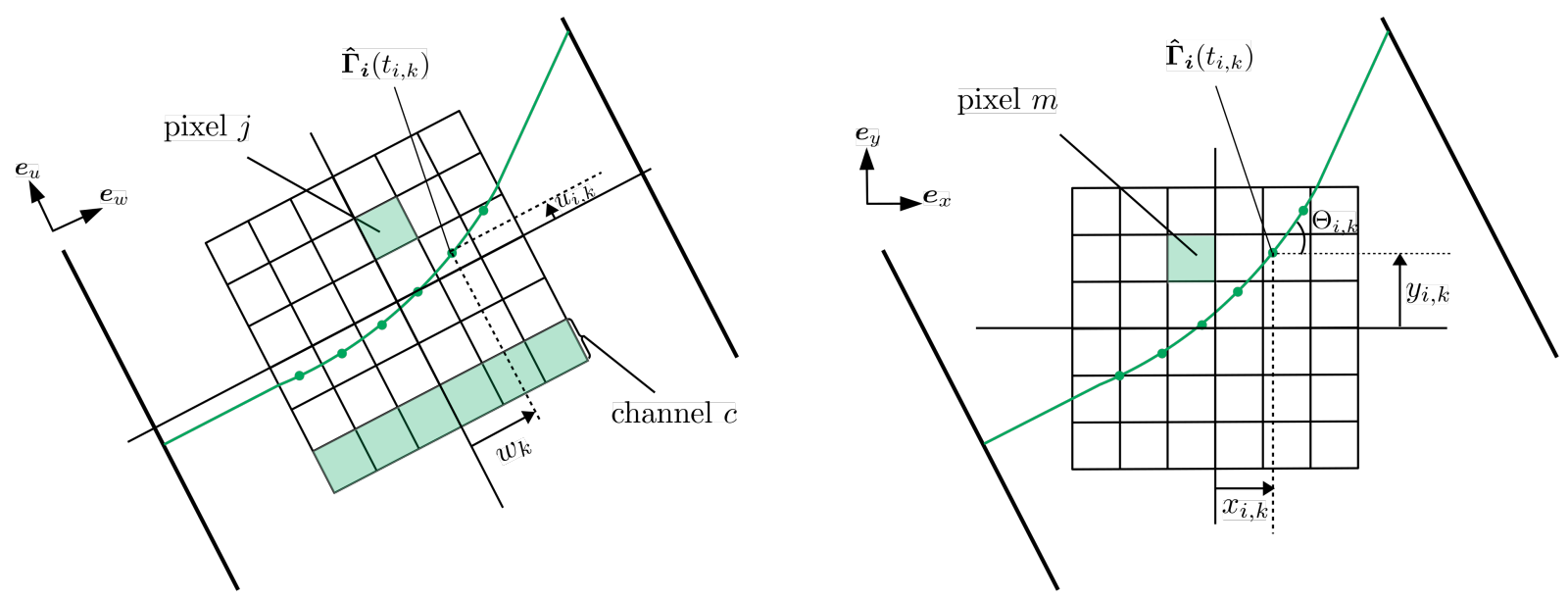

Figure 1: pCT set-up with a parallel proton beam. The left figure corresponds to the geometry in the frame of reference of the source/detector, i.e. the unit vectors $\left(\boldsymbol{e}_{u}, \boldsymbol{e}_{w}\right)$ define a coordinate system relative to the projection angle, with $\boldsymbol{e}_{w}$ the proton beam direction and $\boldsymbol{e}_{u}$ defining the detector surface. The right figure corresponds to the geometry in the frame of reference of the object, with $\left(\boldsymbol{e}_{x}, \boldsymbol{e}_{y}\right)$ a fixed coordinate system. The MLP is computed for each proton $i$ at each $w_{k}$ giving $u_{i, k}$ and $v_{i, k}$ (the axial coordinate is not represented on this $2 \mathrm{D}$ slice). $x_{i, k}$ and $y_{i, k}$ correspond to a rotation of $w_{k}$ and $u_{i, k}$.

\subsection{Reconstruction algorithms}

The following sections summarize the five algorithms included in this comparison. All algorithms are presented in a parallel beam set-up for simplicity, although they can all be used with other geometries.

\subsubsection{Distance-driven algorithm (DD)}

It is the first direct reconstruction algorithm incorporating the MLP that was proposed in the literature (Rit et al., 2013). It consists in binning the protons into projections on a series of planes parallel to the detectors (Figure 1) according to the estimated proton path $\hat{\boldsymbol{\Gamma}}_{i}(t) \in \mathbb{R}^{3}$ at time $t \in \mathbb{R}$, rather than binning just in the detector plane. The projection binning is done at several distances, hence the name of the algorithm.

The MLP formalism yields the coordinates $u_{i}(w)$ and $v_{i}(w)$ for each proton $i$ (Figure 1). In practice, we compute this trajectory at a discrete number of positions $k \in\{1, \ldots, K\}$ such that $u_{i, k}=u_{i}\left(w_{k}\right)$ and $v_{i, k}=v_{i}\left(w_{k}\right)$ with $w_{k}$ the discrete distances. The $3 \mathrm{D}$ proton trajectory at the time when proton $i$ crosses the plane parallel to the detectors at distance $w_{k}$ is written $\hat{\boldsymbol{\Gamma}}_{i}\left(t_{i, k}\right) \in \mathbb{R}^{3}$. Without loss of generality, we use the 3D path to bin the list-mode data into projections, but only the $2 \mathrm{D}$ central slice is considered in the following. The value in the sinogram for pixel $j$ and source position $p$ is given by

$$
g_{j, p}=\frac{\sum_{i \in \mathbb{I}_{p}} \sum_{k} \zeta_{j}\left(u_{i, k}, v_{i, k}, w_{k}\right) \mathrm{WEPL}_{i}}{\sum_{i \in \mathbb{I}_{p}} \sum_{k} \zeta_{j}\left(u_{i, k}, v_{i, k}, w_{k}\right)}
$$

where $\zeta_{j}$ is the indicator function for pixel $j$ defined as

$$
\zeta_{j}(\boldsymbol{y})= \begin{cases}1 & \text { if } \boldsymbol{y} \in \mathbb{R}^{3} \text { is in pixel } j, \\ 0 & \text { otherwise }\end{cases}
$$

and $\mathbb{I}_{p}$ is the subset of protons emitted from the same source position $p$. Note that the sinogram for a single slice is in $3 \mathrm{D}$ since it depends on the source position and the $2 \mathrm{D}$ pixel index. 
The filtering and backprojection steps are the same as in a standard filtered backprojection algorithm, except that a voxel-specific backprojection is used to select which distance $w$ to use, instead of the usual pixel-specific backprojection. Specifically, we define the $2 \mathrm{D}$ projections $g_{p}(u, w): \mathbb{R}^{2} \rightarrow \mathbb{R}$ as

$$
g_{p}(u, w)=\sum_{j} g_{j, p} \psi_{j}(u, w),
$$

with $\psi_{j}(u, w)$ a given interpolation function. The reconstructed image $f^{\mathrm{DD}}(\boldsymbol{x}): \mathbb{R}^{2} \rightarrow \mathbb{R}$ is then given by

$$
f^{\mathrm{DD}}(\boldsymbol{x})=\sum_{p} \tilde{g}_{p}\left(\boldsymbol{x} \cdot \boldsymbol{e}_{u}, \boldsymbol{x} \cdot \boldsymbol{e}_{w}\right) \Delta \theta
$$

with $\boldsymbol{x}=(x, y) \in \mathbb{R}^{2}, \tilde{g}_{p}$ the ramp-filtered projection $g_{p}$ in the case of a parallel beam geometry, and $\Delta \theta$ the constant angular spacing between consecutive projections.

\subsubsection{Maximum likelihood method (ML)}

This method proposes to maximize the likelihood of the water equivalent thickness (WET) in proton radiographs, i.e. on the projection level, in order to improve their spatial resolution (Collins-Fekete et al., 2016). These optimized radiographs are then used to reconstruct a pCT image. Specifically, the WET's likelihood is maximized for each so-called "channel" $c$ in a radiograph, where a channel is the volume defined by the pixel corners along the source direction (see Figure 1). The WET value for channel $c$ and source position $p$ is obtained given the measured WEPL and the estimated trajectory for each proton using

$$
\mathrm{WET}_{c, p}=\frac{\sum_{i \in \mathbb{I}_{p}} \frac{l_{c, i}^{2}}{L_{i}^{2}} \mathrm{WEPL}_{i}}{\sum_{i \in \mathbb{I}_{p}} \frac{l_{c, i}^{2}}{L_{i}^{2}}}
$$

with $l_{c, i}$ the length of the $i$-th proton in channel $c$ and $L_{i}$ its total path length. This is similar to the DD algorithm, except that the WEPL is integrated along the $w$ direction to form a single projection per source position while the DD uses a series of depth dependent projections. Also, the weights for the ML correspond to proportions of proton trajectories spent in a given channel $\left(\frac{l_{c, i}^{2}}{L_{i}^{2}}\right)$ while for the DD method, the weighting function $\zeta_{j}$ is binary. To reconstruct the pCT image, the radiographs $\mathrm{WET}_{c, p}$ are simply fed to a standard filtered backprojection algorithm (Feldkamp et al., 1984). As in the DD, we define interpolated projections

$$
\operatorname{WET}_{p}(u)=\sum_{c} \operatorname{WET}_{c, p} \psi_{c}(u),
$$

giving the following reconstruction

$$
f^{\mathrm{ML}}(\boldsymbol{x})=\sum_{p} \widetilde{\mathrm{WET}}_{p}\left(\boldsymbol{x} \cdot \boldsymbol{e}_{u}\right) \Delta \theta
$$

where $\widetilde{\mathrm{WET}}_{p}(u)$ is the filtered projection $\operatorname{WET}_{p}(u)$

\subsubsection{Backprojection-then-filtering (BTF)}

This algorithm inverts the order of the backprojection and the filtering so that we directly backproject the protons along their MLPs, instead of binning them into a sinogram first (Polud- 
niowski et al., 2014). An intermediate step for calculating the backprojection from the list-mode data is a pixel-wise and direction-wise binning,

$$
b_{m, n}=\frac{\sum_{i, k} \zeta_{m}\left(\hat{\boldsymbol{\Gamma}}_{i}\left(t_{i, k}\right)\right) \xi_{n}\left(\Theta_{i, k}\right) \mathrm{WEPL}_{i}}{\sum_{i, k} \zeta_{m}\left(\hat{\boldsymbol{\Gamma}}_{i}\left(t_{i, k}\right)\right) \xi_{n}\left(\Theta_{i, k}\right)},
$$

with indicator functions

$$
\zeta_{m}\left(\hat{\boldsymbol{\Gamma}}_{i}\left(t_{i, k}\right)\right)= \begin{cases}1 & \text { if } \hat{\boldsymbol{\Gamma}}_{i}\left(t_{i, k}\right) \in \mathbb{R}^{3} \text { is in pixel } m \\ 0 & \text { otherwise }\end{cases}
$$

and

$$
\xi_{n}\left(\Theta_{i, k}\right)= \begin{cases}1 & \text { if } \Theta_{i, k} \in \mathbb{R} \text { is in angular bin } n . \\ 0 & \text { otherwise. }\end{cases}
$$

This binning was first presented by Rit et al. (2015). The WEPL for each proton is smeared along the corresponding path in the image grid using $\zeta_{m}$, an indicator function for pixel $m$, and $\xi_{n}$, an indicator function for angular bin $\theta_{n}$. The angle $\Theta_{i, k}$ is that of proton $i$ at depth $w_{k}$ with respect to $\boldsymbol{e}_{x}$ (Figure 1). This binning was also used for the algorithms described in sections 2.2.4 and 2.2.5. Note that $b_{m, n}$ is not strictly speaking a backprojection but rather an average of the WEPL of protons passing through pixel $m$ with an angle $\simeq \theta_{n}$. This direction-wise binning allows to rebin the data into parallel geometry (in case another geometry is used) as it uses the proton's angle $\Theta_{i, k}$ with respect to $\boldsymbol{e}_{\boldsymbol{x}}$ rather than the subsets $\mathbb{I}_{p}$ as in the previous methods. We interpolate an image $b_{n}(\boldsymbol{x}): \mathbb{R}^{2} \rightarrow \mathbb{R}$ defined as

$$
b_{n}(\boldsymbol{x})=\sum_{m} b_{m, n} \psi_{m}(\boldsymbol{x})
$$

giving the backprojected image

$$
B(\boldsymbol{x})=\sum_{n} b_{n}(\boldsymbol{x}) \Delta \theta
$$

In the BTF method (Poludniowski et al., 2014), the image is reconstructed by convolving the backprojection with a $2 \mathrm{D}$ cone filter $c(\boldsymbol{x})$, yielding

$$
f^{\mathrm{BTF}}(\boldsymbol{x})=(B * c)(\boldsymbol{x}) .
$$

The expression for the filter $c(\boldsymbol{x})$ is given by Poludniowski et al. (2014) in Equations (12)-(14). It was derived by taking the inverse Fourier transform of the cone filter $\rho=\left\|\left(\nu_{x}, \nu_{y}\right)\right\|$ where $\left(\nu_{x}, \nu_{y}\right)$ are the $2 \mathrm{D}$ Fourier variables.

The backprojection $B$ has an infinite support. In order to mitigate the effects of truncating $B$ during the convolution, the backprojection is computed on a very large matrix (4 to 8 times the size of the reconstructed image). We chose to use a factor of 4 to keep a reasonable computation time. The contribution of distant data of $B$ to the central pixel is approximated by a factor $\Delta f$ (Equation 19 (Poludniowski et al., 2014)) obtained by replacing $B=f^{\mathrm{BTF}} * 1 / r$ in Equation 14 when $r \rightarrow \infty$, with $r=\|(x, y)\|$. This factor is used to correct for the constant bias (i.e. pixel independent) induced by the truncation of the backprojection matrix, giving the final image

$$
f^{\mathrm{BTF}, \Delta}=f^{\mathrm{BTF}}+\Delta f .
$$




\subsubsection{Directional Ramp filtering (DR)}

This is an intermediate method between a backproject-first and a filter-first algorithm (Khellaf et al., 2020). It starts by (1) computing the pixel-wise and direction-wise binning (Equation 9), then (2) filtering each direction with the corresponding directional ramp filter and (3) summing all filtered backprojections. The reconstructed image is given by

$$
f^{\mathrm{DR}}(\boldsymbol{x})=\sum_{n} \tilde{b}_{n}(\boldsymbol{x}) \Delta \theta
$$

where

$$
\tilde{b}_{n}(\boldsymbol{x})=\left(b_{n} * h_{n}\right)(\boldsymbol{x}) .
$$

The expression of $h_{n}$ is given by (Khellaf et al., 2020). The directional filter is a two-dimensional version of the usual ramp filter. As in backproject-first algorithms, this method starts by smearing the projections into the image space before filtering, but the sum over all projection angles is the last step, as in filter-first methods.

\subsubsection{Differentiated backprojection (DBP)}

This algorithm is based on the two-step Hilbert transform method (Noo et al., 2004). It relies on the relationship between the backprojection of the derivative of the projections and the Hilbert filtered image:

$$
f(x, y)=\left.\mathcal{H}^{-1} \int_{0}^{\pi} \frac{\partial}{\partial s} p(s, \theta)\right|_{s=-x \sin \theta+y \cos \theta} \mathrm{d} \theta,
$$

with $\mathcal{H}^{-1}$ the inverse of the Hilbert transform. Noo et al. (2004) give a formula to invert the finite Hilbert transform, which requires knowledge of the convex hull of the object (an information which is also used in our implementation of the MLP). Simply put, the image can be reconstructed by inverting the finite Hilbert transform of the differentiated backprojection. The advantage of this approach is that, since the derivative is a local operator, truncated data can still be reconstructed in cases when points are lying on at least one line segment having its two end points in the field of view and outside the object to reconstruct. This method was adapted to work with list-mode data by inverting the order of the differentiation and the backprojection steps (Zeng, 2007), giving the following reconstruction formula

$$
f^{\mathrm{DBP}}(x, y)=\mathcal{H}^{-1}\left(\frac{\partial}{\partial x} B^{s}(x, y)+\frac{\partial}{\partial y} B^{c}(x, y)\right) .
$$

Due to the partial differentiation, two intermediate weighted backprojections must be computed

$$
\begin{aligned}
B^{s}(\boldsymbol{x}) & =-\sum_{n} b_{n}(\boldsymbol{x}) \sin \theta_{n} \Delta \theta, \\
B^{c}(\boldsymbol{x}) & =\sum_{n} b_{n}(\boldsymbol{x}) \cos \theta_{n} \Delta \theta .
\end{aligned}
$$

This method was applied to pCT data by Rit et al. (2015).

\subsection{Simulations}

Monte Carlo simulations were conducted using Geant4 (Agostinelli et al., 2003) through Gate (Jan et al., 2011) to acquire pCT list-mode data. A "single tracking" setup was used, consisting of two ideal detectors measuring position, direction and energy, located upstream and downstream from the phantoms. A $200 \mathrm{MeV}$ rotating fan-beam proton source was positioned at $1 \mathrm{~m}$ distance from the object center, with a flux of 225 protons $\cdot \mathrm{mm}^{-2}$.projection ${ }^{-1}$ at the isocenter, for a total of 720 projections over a 360 degree range. Protons were paired between the two detectors, 


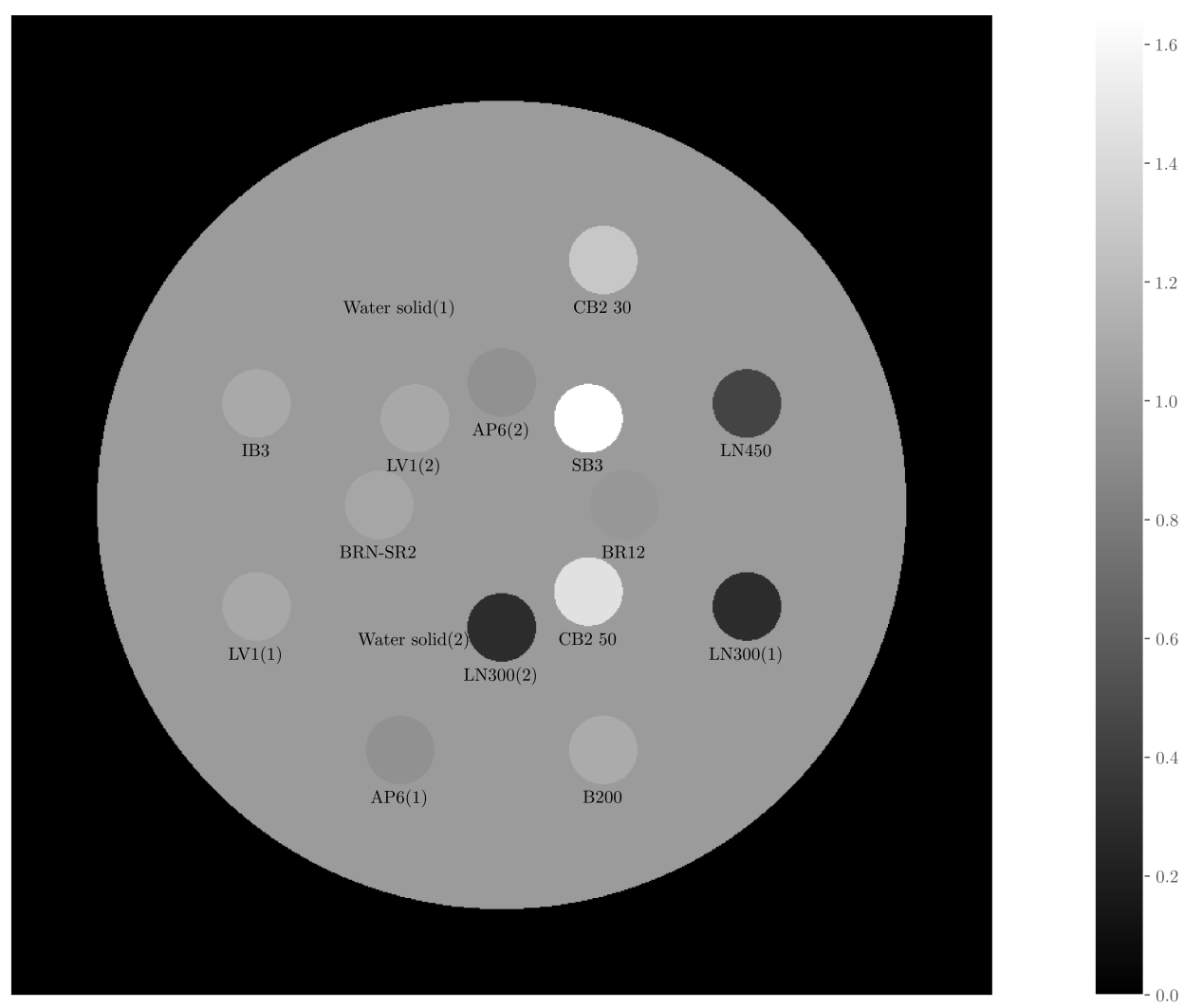

Figure 2: RSP map of the Gammex 467 tissue characterization phantom.

and each proton's path was estimated using the MLP formalism, after excluding protons with nuclear interactions using $3 \sigma$ angle and energy cuts (Schulte et al., 2008).

Three phantoms were imaged: the CTP528 module of the Catphan phantom (The Phantom Laboratory, NY) with aluminium line pairs of different resolutions in a $20 \mathrm{~cm}$ diameter water cylinder; a spiral phantom, consisting of aluminium cylinders placed along a spiral in a $20 \mathrm{~cm}$ diameter water cylinder (Rit et al., 2015), and the Gammex 467 tissue characterization phantom with different tissues inserts inside a $33 \mathrm{~cm}$ diameter water cylinder (Figure 2). The phantoms used for spatial resolution assessment (Catphan and spiral) were reconstructed in images of $1000 \times 1000$ pixels with a pixel spacing of $0.25 \mathrm{~mm}$. The Gammex 467 was reconstructed on a grid of $800 \times 800$ pixels of spacing $0.5 \mathrm{~mm}$ and required a beam energy of $250 \mathrm{MeV}$ because of its larger diameter.

In order to estimate the impact of detector uncertainties on spatial resolution, an uncertainty on the simulated position and direction data was added using the formalism of Krah et al. (2018). The parameters were chosen to reflect a typical pCT prototype. In particular, the detector resolution (pitch of strips) was characterized by a Gaussian of $\sigma_{p}=0.15 \mathrm{~mm}$, the distance between the trackers was set to $d_{T}=10 \mathrm{~cm}$ (each real detector consists of a pair of trackers), the distance between the trackers and the object border to $20 \mathrm{~cm}$, and the material budget to $x / X_{0}=5 \times 10^{-3}$.

The energy resolution of the detectors and the spread of the incident beam were omitted for simplicity as scintillating detectors (Bashkirov et al., 2016) are capable of achieving a WEPL resolution close to the limit due to proton range straggling (measured WEPL resolution of $3 \mathrm{~mm}$ for a range straggling of $2.85 \mathrm{~mm}$, for $200 \mathrm{MeV}$ protons in water), although we note the beam spread can contribute to the WEPL variance up to 20\% (Dickmann et al., 2019). Electromagnetic interactions are taken into account in the simulation and are responsible for the RSP resolution of the reconstructed images. 


\subsection{Image quality metrics}

Both spatial resolution and RSP accuracy were evaluated. The spatial resolution was assessed qualitatively using the Catphan phantom and quantitatively with the spiral phantom. The modulation transfer function (MTF) was calculated in the spiral phantom using each aluminium cylinder's radial edge-spread-function (ESF), as was done by (Richard et al., 2012). An error function with parameters $\mu$ and $\sigma$ was fitted to sampled points of the ESF, assuming

$$
\operatorname{ESF}(x) \propto \frac{1}{2}\left(1+\operatorname{erf}\left(\frac{x-\mu}{\sigma}\right)\right) .
$$

The frequency corresponding to an MTF value of $10 \%$, given by

$$
\mathrm{MTF}_{10 \%}=\sqrt{\frac{\ln 10}{2}} \frac{1}{\pi \sigma},
$$

was used to quantify the spatial resolution. The error on $\mathrm{MTF}_{10 \%}$ was derived from the standard deviation of the error function fit. In addition, we measured the radial and azimuthal resolutions defined as defined by Plautz et al. (2016) in order to assess the (an)isotropy of the spatial resolution. The radial resolution was measured using ESFs along the direction from the isocenter to the center of the bead and the azimuthal resolution was measured from profiles along the direction perpendicular to the radial direction. Profiles were sampled and averaged for angles in a range of $\pm 10^{\circ}$ around these two directions.

Each algorithm's RSP accuracy was evaluated by computing the mean value in each insert of the Gammex phantom. A region of interest (ROI) of radius $8 \mathrm{~mm}$ was used (the radius of the inserts is $14 \mathrm{~mm}$ ) so that the measured RSP value would not be impacted by the inserts' spatial resolution. The uncertainty on the measured RSP is computed using the $95 \%$ confidence limits of the mean, i.e. $\pm 1.96 \sigma_{\mathrm{ROI}} / \sqrt{N_{\mathrm{ROI}}}$, with $\sigma_{\mathrm{ROI}}$ the standard deviation of the RSP values in the ROI and $N_{\text {ROI }}$ the number of pixels inside the ROI.

\section{RESULTS}

The reconstructed images of the Catphan and spiral phantoms are shown in Figure 3 . For the Catphan phantom, the five reconstructions are quite similar, as confirmed by the line profiles drawn in Figure 4. All algorithms underestimate the RSP of aluminium in the Catphan phantom due to the impact of axial resolution. The ML reconstruction leads to a lower resolution, causing a larger underestimation of the RSP value of the aluminium inserts. Regarding the spiral phantom reconstructed from data simulated with ideal trackers (middle column of Figure 3), the central beads appear much blurrier than the outer beads for all algorithms. This is expected because the MLP uncertainty is largest for those inserts. A slight artifact is seen for the ML reconstruction where the outer beads appear smeared along the azimuthal direction. This is visible on the zoomed insets, although it is exaggerated by using a color scale with a small range. Figure 5 shows the spatial resolution as a function of each bead's distance to the centre of the spiral phantom, for reconstructions with ideal trackers and realistic trackers. For ideal trackers, resolution is about a factor 4 to 6 higher for beads near the phantom borders compared with the resolution at the centre. The resolution of the ML algorithm is lower than other algorithms: it only reaches $1 \mathrm{lp} / \mathrm{mm}$ for the outermost bead and is also smaller for central beads. Differences in spatial resolution among the other four algorithms are much smaller, especially below a distance of about $80 \mathrm{~mm}$ from the center. Beyond that distance, i.e. closer to the phantom edge, the DR and BTF algorithms reach similar resolutions (up to 3.5 and $3.3 \mathrm{lp} / \mathrm{mm}$, respectively), while the DBP and DD methods both reach about $2.7 \mathrm{lp} / \mathrm{mm}$. Figure 6 presents a more extensive analysis of the ideal spatial resolution, discriminating between the radial and azimuthal resolutions. The azimuthal resolution is higher than the radial resolution for all algorithms except ML. 
Table 1: Relative error on the measured RSP values.

\begin{tabular}{lllllll}
\hline Insert & Reference & DD & BTF & DBP & ML & DR \\
\hline LN300(1) & 0.295 & $-0.15 \%$ & $0.02 \%$ & $-0.44 \%$ & $1.32 \%$ & $-0.21 \%$ \\
IB3 & 1.094 & $-0.04 \%$ & $0.02 \%$ & $-0.19 \%$ & $-0.02 \%$ & $-0.03 \%$ \\
LV1 (1) & 1.084 & $-0.03 \%$ & $0.05 \%$ & $-0.21 \%$ & $-0.03 \%$ & $-0.01 \%$ \\
LN450 & 0.443 & $-0.27 \%$ & $-0.06 \%$ & $-0.24 \%$ & $0.54 \%$ & $-0.21 \%$ \\
B200 & 1.103 & $-0.04 \%$ & $0.06 \%$ & $-0.18 \%$ & $-0.17 \%$ & $<0.01 \%$ \\
Water_Solid (1) & 1.005 & $-0.03 \%$ & $0.06 \%$ & $-0.16 \%$ & $0.05 \%$ & $-0.01 \%$ \\
CB2_30 & 1.286 & $0.04 \%$ & $0.10 \%$ & $-0.14 \%$ & $-0.13 \%$ & $0.07 \%$ \\
AP6 (1) & 0.940 & $0.04 \%$ & $0.08 \%$ & $-0.15 \%$ & $0.03 \%$ & $0.02 \%$ \\
BR12 & 0.974 & $-0.01 \%$ & $-0.17 \%$ & $-0.15 \%$ & $-0.03 \%$ & $0.01 \%$ \\
BRN-SR2 & 1.073 & $0.08 \%$ & $-0.06 \%$ & $-0.05 \%$ & $0.01 \%$ & $0.07 \%$ \\
CB2_50 & 1.449 & $-0.11 \%$ & $-0.18 \%$ & $-0.25 \%$ & $-0.13 \%$ & $-0.08 \%$ \\
SB3 & 1.649 & $-0.16 \%$ & $-0.19 \%$ & $-0.20 \%$ & $-0.13 \%$ & $-0.08 \%$ \\
LN300 (2) & 0.295 & $0.05 \%$ & $-0.59 \%$ & $-0.30 \%$ & $1.16 \%$ & $-0.07 \%$ \\
AP6 (2) & 0.940 & $0.14 \%$ & $<0.01 \%$ & $0.19 \%$ & $0.18 \%$ & $0.18 \%$ \\
LV1 (2) & 1.084 & $0.04 \%$ & $-0.08 \%$ & $-0.06 \%$ & $0.06 \%$ & $0.07 \%$ \\
Water_Solid (2) & 1.005 & $0.06 \%$ & $-0.06 \%$ & $-0.11 \%$ & $0.06 \%$ & $0.07 \%$ \\
\hline Mean abs error & & $0.08 \%$ & $0.11 \%$ & $0.19 \%$ & $0.25 \%$ & $0.07 \%$ \\
\hline
\end{tabular}

The right column of Figure 3 shows that the resolution with realistic trackers is considerably lower than with ideal ones. In Figure 5, we observe that the maximum resolution for all algorithms only reaches between 0.39 and $0.42 \mathrm{lp} / \mathrm{mm}$, and that the difference of spatial resolution between the center and the borders is much less important than with ideal detectors. For example, for the DD algorithm, it only increases by a factor of 1.3 between the central bead and the outer bead, versus a factor of 4.6 with ideal detectors. In addition, the differences in spatial resolution observed between the algorithms are mostly insignificant, considering the uncertainty on the MTF. Only the ML algorithm still shows a slightly lower resolution although it is much closer to other algorithms compared with to ideal case.

Table 1 shows the relative error on the RSP value in each insert of the reconstructed Gammex phantom. The most accurate algorithms are the DR and DD, with a mean relative error of $0.07 \%$ and $0.08 \%$ respectively, followed by the BTF with an error of $0.11 \%$, the DBP with $0.19 \%$, and the ML with a mean error of $0.25 \%$. The uncertainty in Figure 7 shows that the majority of the reconstructed RSP values are very close, with differences within the confidence intervals of the mean. The RSP error in the lung inserts, however, reaches over $1 \%$ for the ML method.

\section{DISCUSSION}

The aim of this work was to compare the performance of five direct reconstruction algorithms in terms of spatial resolution and RSP accuracy. Spatial resolution in pCT is impacted by various factors. A first factor is MCS which is mitigated to some extent by modeling the MLP (Schulte et al., 2008). This greatly improves spatial resolution compared to naive straight line trajectories, although the effect of scattering is still important especially at the center of the phantoms where the MLP estimate is most uncertain. We have observed that the resolution at the center of a $20 \mathrm{~cm}$ wide phantom is about $0.5-0.6 \mathrm{lp} / \mathrm{mm}$ with ideal trackers. For the DR method, the resolution is improved by a factor of 6 between the center and the border of the spiral phantom. Since the center of the object corresponds to the largest uncertainty on the MLP, differences in spatial resolution between the algorithms are not significant near the center. Another consequence of proton scatter is the anisotropy of the spatial resolution. As expected, the azimuthal resolution gets higher than the radial resolution with the distance to the center. Due to scatter, the error envelope of the MLP of protons traversing the same region will depend on the thickness of material traversed, i.e. on their initial direction. For central beads, the 


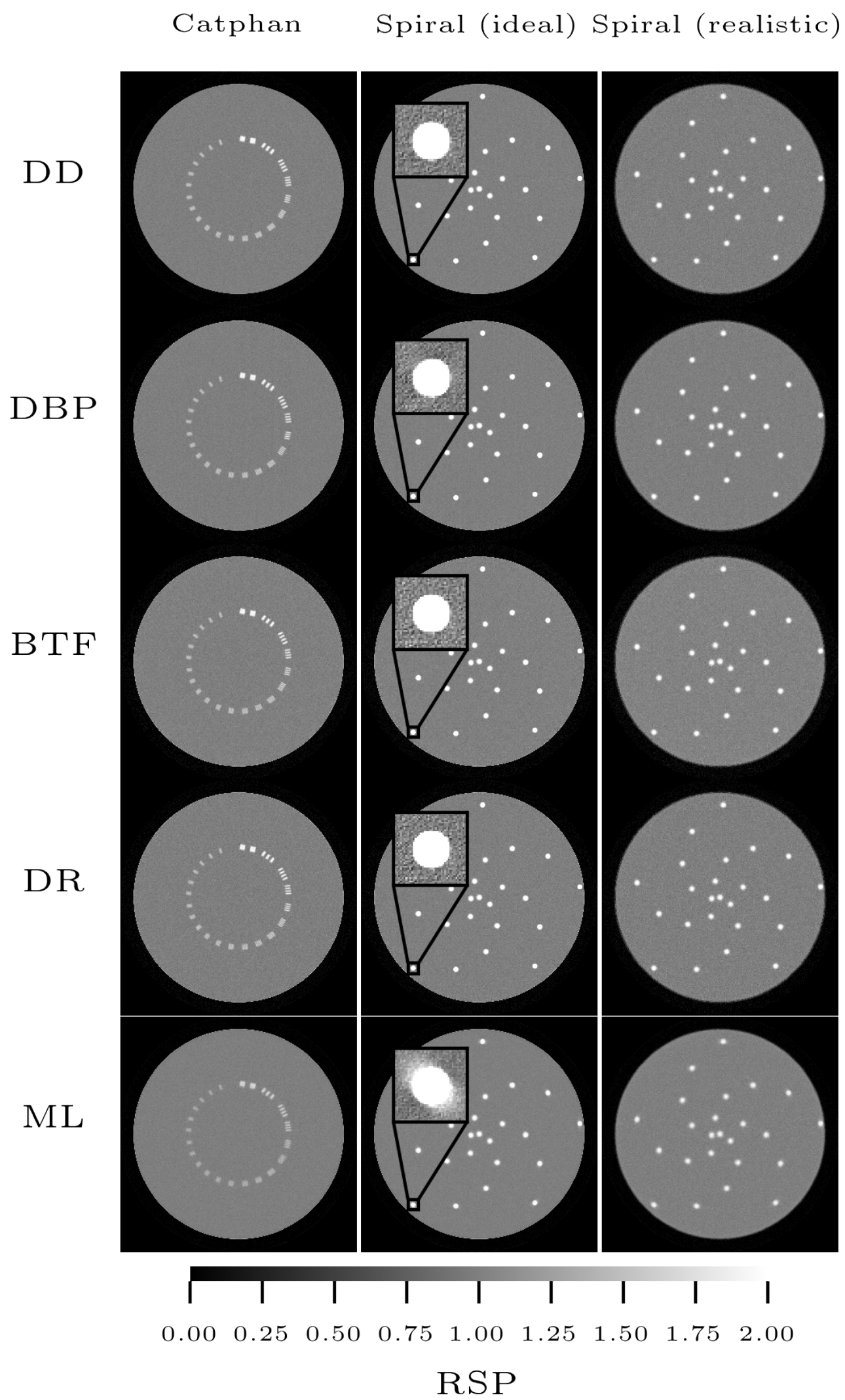

Figure 3: Reconstructions of the Catphan and spiral phantoms for the five algorithms. The Catphan is reconstructed from idealized data. Zoomed insets of a peripheral bead are shown to appreciate the artifact reconstructed by the ML algorithm. The extent of the color scale in the insets is $[0.7,1.3]$ to highlight the artifact. 

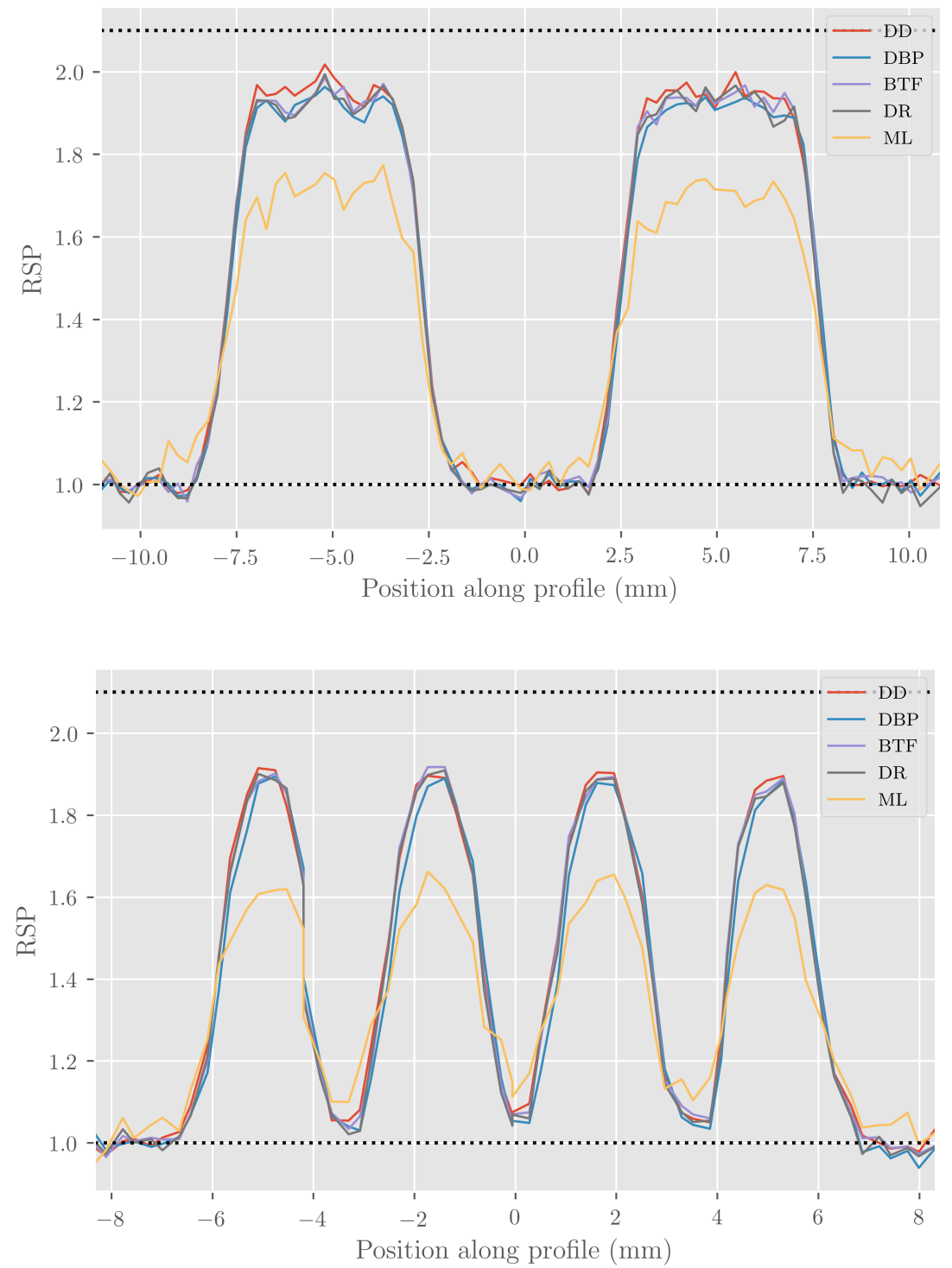

Figure 4: Line profiles along first (top) and third (bottom) line pairs in the Catphan phantom. The dotted lines represent the theoretical $\mathrm{RSP}$ values of water $(\mathrm{RSP}=1)$ and aluminium $(\mathrm{RSP}=2.1)$. 

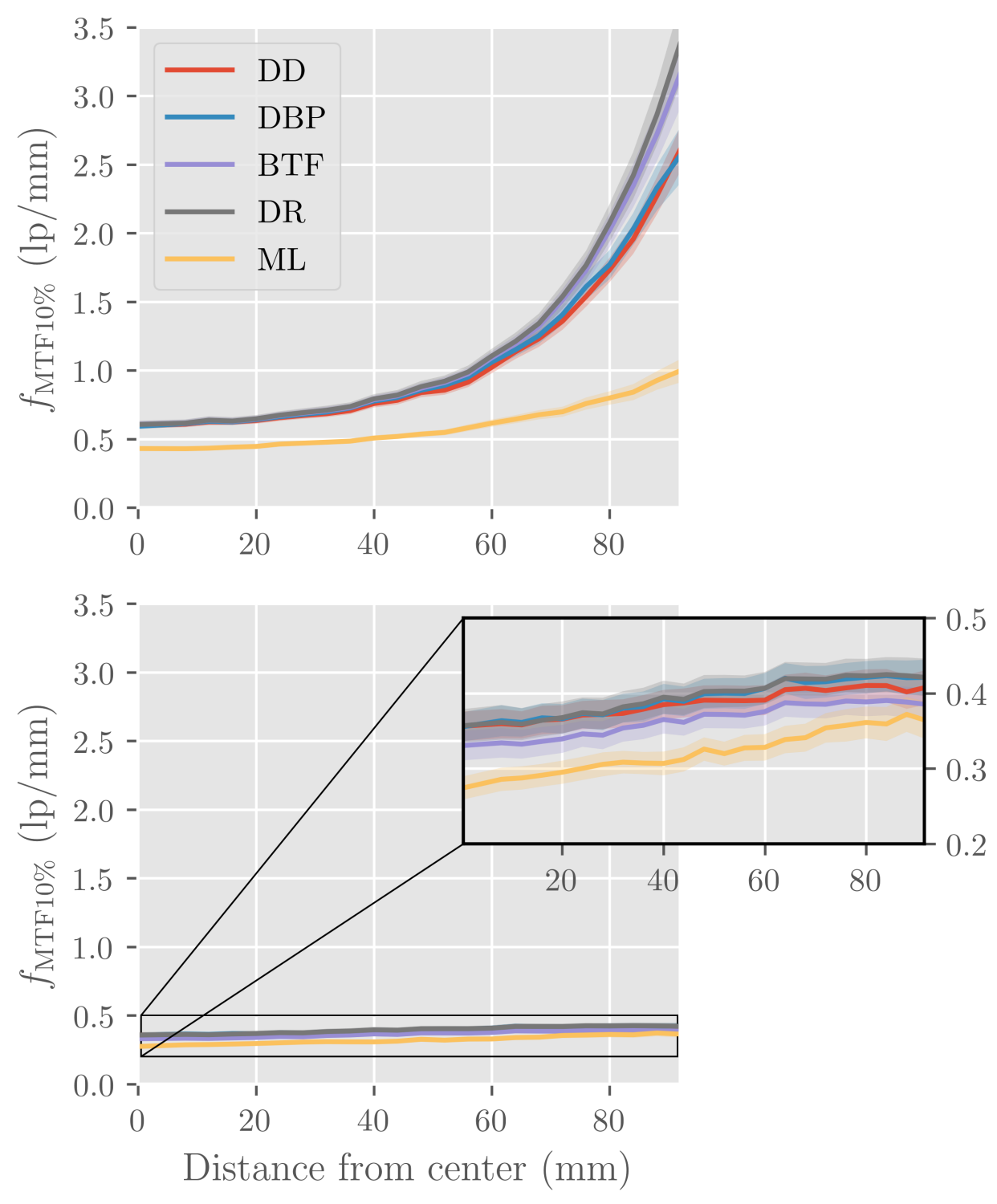

Figure 5: Spatial resolution measured as the frequency for an MTF value of $10 \%$ in function of distance from the isocenter, for ideal trackers (top) and realistic trackers (bottom). The shaded areas represent the uncertainty on the MTF estimation. 


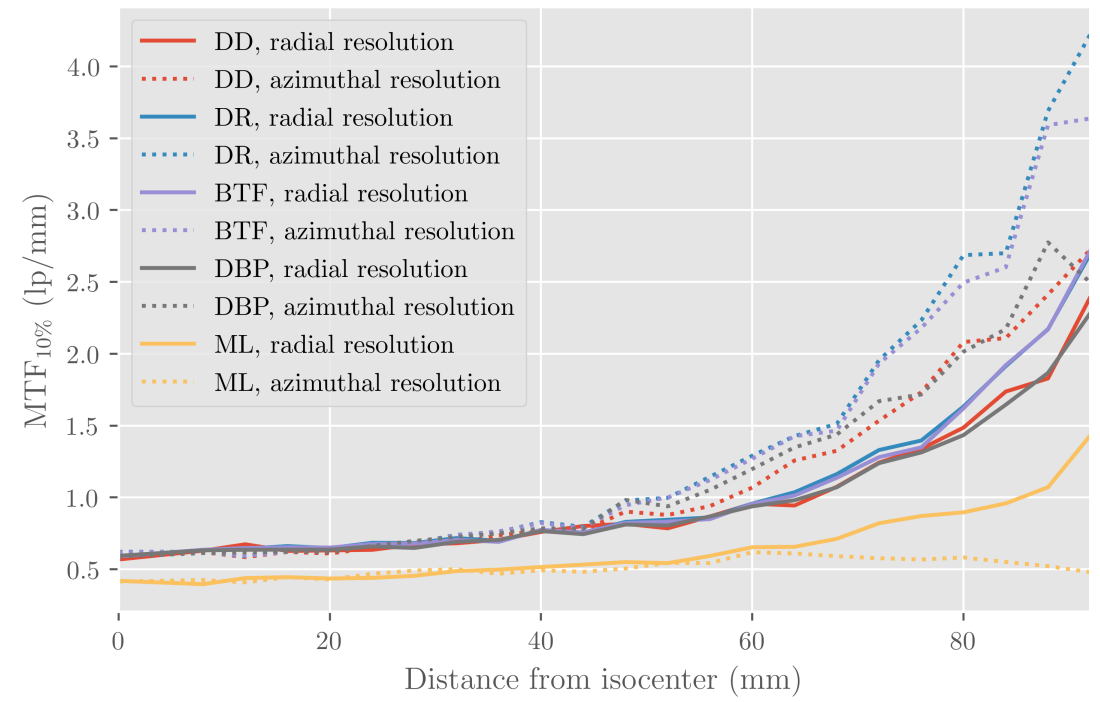

Figure 6: Spatial resolution for ideal trackers computed on profiles along the normal and tangent directions to the edge. Error bars were omitted for clarity.

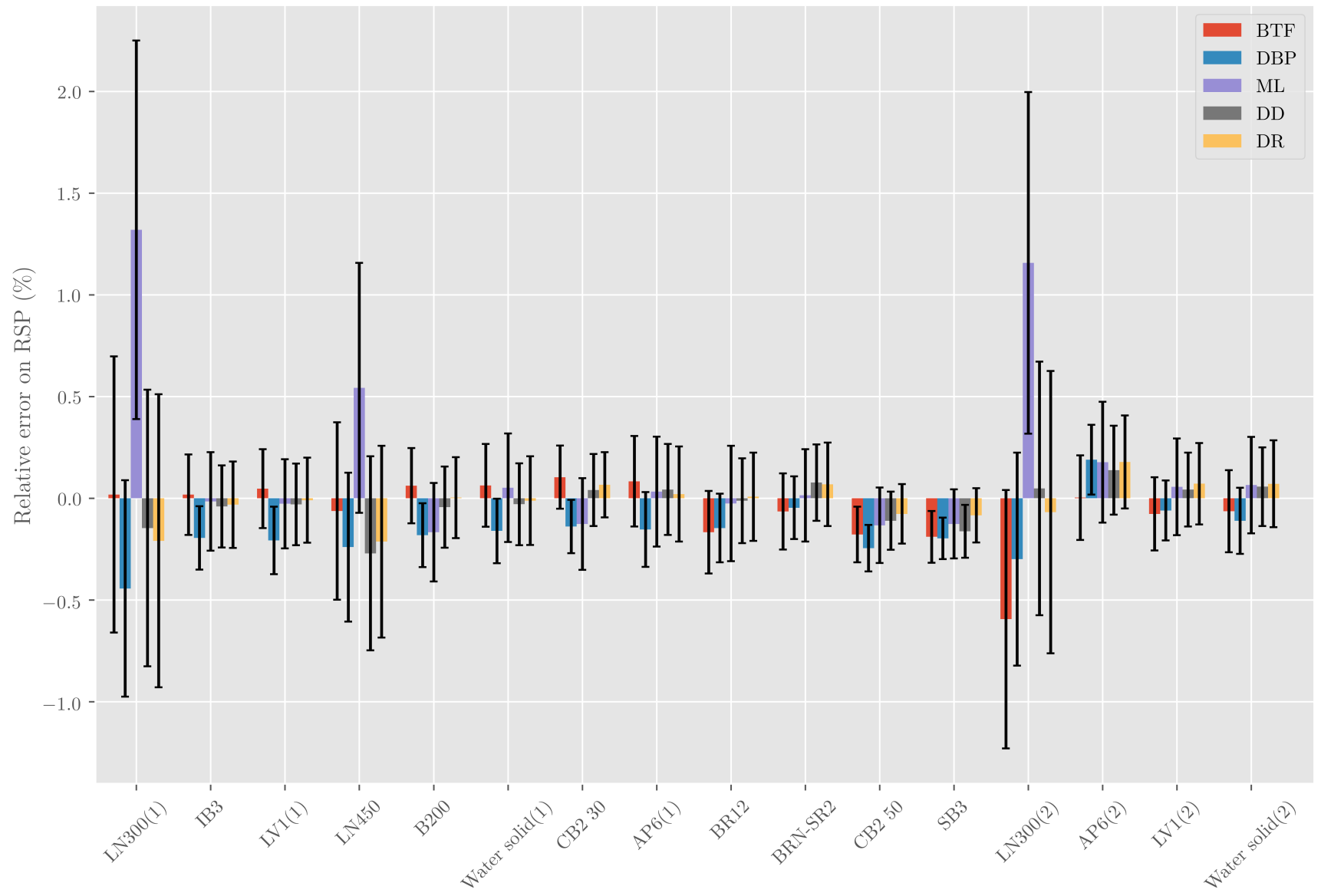

Figure 7: Relative error on RSP with confidence limits. 
variation of the traversed thickness depending on direction will be smaller, such that resolution is isotropic at the center. The only algorithm for which the azimuthal resolution is poor is the ML algorithm, due to the artifact observed in Figure 3. This artifact can be explained by the uncertainty on the MLP. We mentioned that the ML method basically consists in integrating the DD's binning along the $w$ direction. We know that the projections that are farthest from the detectors along the $w$ direction will be the most blurry due to a large uncertainty on the MLP. For the outer beads of the spiral phantom, the most blurry projection contributing to their reconstruction is the one where protons have had the longest trajectory inside the phantom, i.e. the ones that contribute to the azimuthal resolution. This explains why the outer beads seem smeared on the ML reconstruction.

Another factor of resolution loss is the tracker uncertainties which have a significant impact as can be seen in Figure 5. Adding an uncertainty on the position and direction measurements dramatically lowers the spatial resolution compared to ideal simulated data. It should be noted that those results were obtained for a distance of $20 \mathrm{~cm}$ between the trackers and the borders of the phantom. Using larger distances would further reduce resolution.

Finally, the choice of the algorithm also has an impact on the resolution as different binnings and interpolations are involved. For example, using ideal detectors, the resolution $96 \mathrm{~mm}$ away from the center for the DR method is $250 \%$ higher than the ML algorithm's resolution (only $25 \%$ at the center), and 26\% higher than the DD algorithm's resolution (1\% at the center). Our results show that the best performing algorithms in terms of spatial resolution away from the center are the DR and BTF, suggesting that backprojecting the protons before filtering can improve spatial resolution. Indeed, filter-first methods imply two interpolations that can reduce spatial resolution: the projection binning and the backprojection. On the other hand, only one is needed for backproject-first methods. This could preserve the spatial information of proton paths and therefore the resolution. However, we have seen that the DBP method, which starts with the backprojection, has the same spatial resolution as the DD. A possible explanation is that the discrete differentiation computed in the DBP algorithm, using differences of adjacent pixels, may cause a loss of spatial resolution. The ML algorithm shows the worst spatial resolution as it attempts to account for MLPs in the 2D projections, but they can only be described in $3 \mathrm{D}$ space. This method might be better suited for proton radiography than CT. Overall, the impact of the choice of the algorithm is negligible compared with MLP and tracker uncertainty.

In terms of RSP, the DR, DD and BTF show good accuracy with mean absolute errors close to $0.1 \%$. The DBP method is slightly less accurate, which can be linked to the computation of a constant during the Hilbert transform inversion (Noo et al., 2004). However, this is not significant with respect to the $95 \%$ confidence interval of the mean (Figure 7). The ML algorithm overestimates the RSP value in the lung inserts compared with the other methods, resulting in a higher mean error. An explanation is that as it uses channels instead of pixels, the WEPL value of a proton that has not traversed a lung insert can still be attributed to it. While this would happen for all inserts, lung inserts would be more impacted because the difference between the RSP of the lung and the rest of the phantom is larger than for other inserts.

The computational cost of each algorithm is an important factor when several algorithms reach similar levels of resolution and RSP accuracy. The binning of the list-mode data is the most costly operation as the MLP of each proton has to be computed. The binning steps to compute projections as in Equation 2 or smeared projections as in Equation 9 are mostly similar in terms of computational cost. This cost increases with the number of protons and the number of evaluations of the MLP. As we have used a high fluence $\left(288 \times 10^{6}\right.$ protons in total $)$ and a small spacing for the MLP evaluation ( $0.25 \mathrm{~mm}$ or $0.5 \mathrm{~mm}$ depending on the phantom), our binning is orders of magnitude slower than the other reconstruction steps (for the DD, $8 \mathrm{~s} /$ projection $\times$ 720 projections for the binning, and 42s for the filtering and backprojection). Both the BPF and DR methods require the backprojection region to be larger than the reconstructed image region, increasing the computation time of the binning. While the DR method requires backprojecting 
the list-mode data in a region larger by a factor of 2 , the BPF method needs a matrix of at least four times the reconstruction matrix. They are therefore the most computationally expensive methods. The cost of the filtering step varies a lot depending on the algorithm. For example, for the DD, a 3D sinogram needs to be filtered (for each slice) compared with the usual 2D sinogram for the ML. However, as we stated above, the cost of the filtering step is quite negligible compared with the cost of the binning step.

\section{CONCLUSION}

We have studied the spatial resolution and RSP accuracy of five different algorithms used to reconstruct proton list-mode data. The results show that, using ideal trackers, two of the methods that bin the list-mode data directly in the image space (DR and BTF) offer a better spatial resolution at the borders of the object, with a maximum of $3.5 \mathrm{lp} / \mathrm{mm}$ at the borders of a $20 \mathrm{~cm}$ thick object. However, considering realistic measurement uncertainties, the impact of the choice of the algorithm on spatial resolution becomes negligible. Furthermore, the DR, DD, and BTF methods show the best RSP accuracy with a mean error close to $0.1 \%$. While the DR and BTF methods have a slight advantage regarding spatial resolution as well as a good accuracy, they are also the most computationally expensive. For faster reconstructions, the DD algorithm offers the same accuracy and an equivalent spatial resolution considering detector uncertainties, and the DBP, while being somewhat less accurate, can be used for reconstruction with truncated data. The ML, as it works in the projection level, is well suited for proton radiography.

\section{Acknowledgements}

The work of Feriel Khellaf was partially supported by the project DIC20161236452 of the Fondation pour la Recherche Médicale (FRM). The work of Nils Krah was supported by funding from the European Union's Horizon 2020 research and innovation programme under the Marie Sklodowska-Curie grant agreement No 753370. This work was performed within the framework of the SIRIC LYriCAN INCa_INSERM_DGOS_12563 and the LABEX PRIMES (ANR-11-LABX0063) of Université de Lyon, within the program "Investissements d'Avenir" (ANR-11-IDEX0007) operated by the French National Research Agency (ANR).

\section{References}

Agostinelli, S. et al. (2003). "GEANT4 a simulation toolkit". In: Nuclear instruments and methods in physics research section A: Accelerators, Spectrometers, Detectors and Associated Equipment 506.3, pp. 250-303.

Arbor, $\mathrm{N}$ et al. (2015). "Monte Carlo comparison of x-ray and proton CT for range calculations of proton therapy beams". In: Physics in Medicine $\mathcal{E}$ Biology 60.19, p. 7585.

Bashkirov, V. et al. (2016). "Novel scintillation detector design and performance for proton radiography and computed tomography". In: Medical physics 43.2, pp. 664-674.

Collins-Fekete, C.-A. et al. (2016). "A maximum likelihood method for high resolution proton radiography/proton CT". In: Physics in Medicine \& Biology 61.23, p. 8232.

Dickmann, J. et al. (2019). "Prediction of image noise contributions in proton computed tomography and comparison to measurements". In: Physics in Medicine $\&$ Biology 64.14, p. 145016.

Fekete, C.-A. C. et al. (2015). "Developing a phenomenological model of the proton trajectory within a heterogeneous medium required for proton imaging". In: Physics in Medicine 83 Biology 60.13, p. 5071.

Feldkamp, L. et al. (1984). "Practical cone-beam algorithm". In: J Opt Soc Am A 1.6, pp. 612619. 
Gordon, R. et al. (1970). "Algebraic reconstruction techniques (ART) for three-dimensional electron microscopy and X-ray photography". In: Journal of theoretical Biology 29.3, pp. 471481.

Hansen, D. C. et al. (2014a). "Improved proton computed tomography by dual modality image reconstruction". In: Medical physics 41.3, p. 031904.

Hansen, D. C. et al. (2014b). "The image quality of ion computed tomography at clinical imaging dose levels". In: Medical physics 41.11, p. 111908.

Hansen, D. C. et al. (2016). "Fast reconstruction of low dose proton CT by sinogram interpolation". In: Physics in Medicine $\mathcal{G}$ Biology 61.15, p. 5868.

Jan, S et al. (2011). "GATE V6: a major enhancement of the GATE simulation platform enabling modelling of CT and radiotherapy". In: Physics in Medicine 63 Biology 56.4, p. 881.

Johnson, R. P. (2018). "Review of medical radiography and tomography with proton beams". In: Reports on progress in physics 81.1, p. 016701.

Khellaf, F. et al. (2020). "2D directional ramp filter". In: Physics in Medicine 6 B Biology 65.8, $08 \mathrm{NT} 01$.

Krah, N. et al. (2018). "A comprehensive theoretical comparison of proton imaging set-ups in terms of spatial resolution". In: Physics in Medicine E Biology 63.13, p. 135013.

Krah, N. et al. (2019). "Polynomial modelling of proton trajectories in homogeneous media for fast most likely path estimation and trajectory simulation". In: Physics in Medicine $\mathcal{E}^{3}$ Biology 64.19, p. 195014.

Li, T. et al. (2006). "Reconstruction for proton computed tomography by tracing proton trajectories: A Monte Carlo study". In: Medical physics 33.3, pp. 699-706.

Noo, F. et al. (2004). "A two-step Hilbert transform method for 2D image reconstruction". In: Physics in Medicine $\&$ Biology 49.17, p. 3903.

Paganetti, H. (2012). "Range uncertainties in proton therapy and the role of Monte Carlo simulations". In: Physics in Medicine $\mathcal{E}$ Biology 57.11, R99.

Penfold, S. et al. (2009). "A more accurate reconstruction system matrix for quantitative proton computed tomography". In: Medical physics 36.10, pp. 4511-4518.

Penfold, S. et al. (2010). "Total variation superiorization schemes in proton computed tomography image reconstruction". In: Medical physics 37.11 , pp. 5887-5895.

Plautz, T. E. et al. (2016). "An evaluation of spatial resolution of a prototype proton CT scanner". In: Medical physics 43.12, pp. 6291-6300.

Poludniowski, G et al. (2014). "Proton computed tomography reconstruction using a backprojectionthen-filtering approach". In: Physics in Medicine $\&$ Biology 59.24, p. 7905.

Richard, S. et al. (2012). "Towards task-based assessment of CT performance: system and object MTF across different reconstruction algorithms". In: Medical physics 39.7, pp. 4115-4122.

Rit, S. et al. (2013). "Filtered backprojection proton CT reconstruction along most likely paths". In: Medical physics 40.3, p. 031103.

Rit, S. et al. (2015). "List-mode proton CT reconstruction using their most likely paths via the finite Hilbert transform of the derivative of the backprojection". In: The 13th International Meeting on Fully Three-Dimensional Image Reconstruction in Radiology and Nuclear Medicine, pp. 324-327.

Schaffner, B et al. (1998). "The precision of proton range calculations in proton radiotherapy treatment planning: experimental verification of the relation between CT-HU and proton stopping power". In: Physics in Medicine \& Biology 43.6, p. 1579.

Schulte, R. et al. (2008). "A maximum likelihood proton path formalism for application in proton computed tomography". In: Medical physics 35.11, pp. 4849-4856.

Williams, D. (2004). "The most likely path of an energetic charged particle through a uniform medium". In: Physics in Medicine \& Biology 49.13, p. 2899. 
Yang, M. et al. (2012). "Comprehensive analysis of proton range uncertainties related to patient stopping-power-ratio estimation using the stoichiometric calibration". In: Physics in Medicine 83 Biology 57.13, p. 4095.

Zeng, G. L. (2007). "Image reconstruction via the finite Hilbert transform of the derivative of the backprojection". In: Medical physics 34.7, pp. 2837-2843. 